\title{
Clasificación motivacional de los nombres de pila basada en la relación estructura-referente
}

\author{
Motivational Classification of First Names Based \\ in the Structure-Reference Relation
}

\author{
Luis Ramón Campo Yumar \\ Universidad Central Marta Abreu de Las Villas \\ lcampo@uclv.cu \\ Evelyn Linet Rabelo Fresno \\ Universidad Central Marta Abreu de Las Villas \\ erabelo@nauta.cu
}

\section{Resumen}

Dentro de las múltiples líneas de investigación que tienen como objeto de estudio el nombre propio de persona, una de las menos estudiadas es la referida a las motivaciones presentes en la selección del nombre de pila. Determinados factores que influyen en la recolección de los datos y la imposibilidad de conocer las razones verdaderas de la elección a partir de las fuentes más accesibles complejizan estos estudios. En el presente trabajo se profundiza en la entrevista y el cuestionario, dentro de las múltiples fuentes, como vías fundamentales para la obtención de datos sobre las motivaciones y referentes utilizados. Como resultado de investigaciones previas y de las consideraciones planteadas en el presente artículo se propone una clasificación motivacional de los nombres de pila basada en la relación estructura - referente. Esta clasificación se aplicó a un pequeño corpus de nombres obteniéndose resultados favorables.

Palabras Clave: antroponimia, nombres de pila, clasificación motivacional, referentes

\section{Abstract}

Among the multiple lines of research that have as their object the name of a person, one of the least studied is the one related to the motivations present in the selection of the first name. Certain factors influencing data collection and the inability to know the true reasons for the choice from the most accessible sources complicate these studies. In the present work, the interview and the questionnaire are explored, within the multiple sources, as fundamental ways to obtain data on the motivations and references used. As a result of previous research and the considerations raised in this 
article, a motivational classification of first names based on the structure - referent relationship is proposed. This classification was applied to a small corpus of names, obtaining favorable results.

KEYWORDS: anthroponymy, first names, motivational classification, references

FECHA DE RECEPCIÓN: 01/06/2020

FECHA DE ACEPTACIÓN: 18/09/2020

\section{Introducción}

Una de las áreas relativas al nombre de pila ${ }^{1}$ que aún sigue despertando el interés de sociólogos, historiadores, lingüistas, juristas, entre otros muchos investigadores es la vinculada a los motivos de su selección. Estas razones e influencias que actúan en los padres y otros familiares dentro de un marco regulatorio ${ }^{2}$ provienen de diferentes fuentes; sin embargo, existen motivos y tipos de referentes universales.

En 1970 al referirse a los motivos de los padres mexicanos para colocarles los nombres a sus hijos el investigador BoydBowman apunta:

Creemos percibir en la onomástica mexicana del siglo xx una progresiva disminución, más rápida en la masculina que en la femenina, del fervor religioso que movía a los padres de recién nacidos en siglos anteriores. Algunos nombres femeninos sí lo manifiestan todavía, pero son cada vez más numerosos los que parecen haberse escogido, no por razones religiosas, sino simplemente por la eufonía, el gusto por lo exótico u original, o por hacer honor a un célebre artista o figura política. Poco a poco, la onomástica mexicana se va secularizando (1970, p. 31).

\footnotetext{
${ }^{1}$ Aunque existen numerosas denominaciones para referirse a este elemento: prenombre, nombre propio de persona, antropónimo, etc. En el presente artículo se utiliza nombre de pila para evitar confusiones y en consonancia con lo planteado por la Real Academia Española: "en el sistema español, los antropónimos están formados por el nombre de pila (que puede ser simple o compuesto) y la mención del primer apellido de cada uno de los progenitores, por este orden: María Rosa Ordóñez Robles; Pedro Linares Cabo" (2010, p. 625).

${ }^{2}$ Sobre la variación geográfica y temporal de las leyes que regulan la imposición del nombre de pila en varias países de Europa y América véase Campo, 2020.
} 
Motivos semejantes son identificados décadas después en España por investigadoras diferentes. Para Hana Kohoutková:

Actualmente existen varios otros motivos para llamar a un recién nacido con cierto nombre: si el nombre es popular entre la gente, si a los padres les gusta algún personaje famoso (actor, actriz, cantante, político) o también si el nombre ya está o no en la familia" (2009, p. 20).

De igual manera, Consuelo García Gallarín (2014) sostiene la prevalencia de determinados motivos en el tiempo y la incorporación de otros, nociones presentes en Boyd-Bowman (1970):

Los motivos no difieren demasiado en la sociedad actual, a diferencia de las costumbres de antaño, los electores del siglo XXI han integrado en su particular enciclopedia de nombres propios los llamados tradicionales, casi todos hagiónimos o advocaciones marianas, pero también nombres de personajes históricos sin reconocimiento eclesiástico, o nombres inspirados en realidades no personales, frecuentemente elementos de la naturaleza (nombres de ríos, lugares, flores, piedras preciosas, etc. $)^{3}$ o abstractos (García Gallarín, 2014, p. 13).

En el Caribe, específicamente República Dominicana y Puerto Rico, se añaden, a los referentes ya abordados, otros vinculados a las características naturales específicas de estas regiones:

En primer lugar se encuentra el santoral católico, que contribuye con los nombres más frecuentes de todo el corpus: María, Altagracia, Carmen, Mercedes, Margarita, Josefina, Isabel, Juana, Ramona, Francisca. Otras fuentes, que pueden ser consideradas secundarias por su menor aportación cuantitativa, son la toponimia y la flora. Dentro de estas categorías aparecen nombres geográficos, como Argentina, Francia, Venecia, Argelia; y de flores, como Magnolia, Orquídea (Alba, 2013, p. 14).

\footnotetext{
${ }^{3}$ Todas las acotaciones y destacados dentro de las citas textuales utilizando cualquier símbolo corresponden a cada uno de los autores del texto citado.
} 
En Puerto Rico, la arena, el mar, el coral, la Luna y el Sol han servido de inspiración al momento de crear nombres, siendo Arenamar, Aurimar, Coralymar, Lunamar, Lunisol y Solymar, algunos ejemplos. No resultaría descabellado pensar que la belleza de la zona tropical caribeña es uno de los criterios de estética que se han adoptado en Puerto Rico al momento de crear un nombre de pila [...] la creación de nombres inspirada en animales no es común, pero se han podido identificar los siguientes nombres: Biajaní (ave), Crisálida (insecto), Ibis (ave) y Coquí (batracio) (Martínez Nazario, 2016, p. 50).

Otros investigadores (Águila, 2014; Alfaro, 2010; Arteaga y Cova, 2003; Ballester, 1999; Carretero Melo, 2001; Fernández Juncal, 2011; Garaio Mendizabal, 2017, entre otros) han abordado las motivaciones presentes en la elección del nombre propio de persona ya sea como centro de sus investigaciones o de forma indirecta. No obstante, Consuelo García Gallarín resume con gran precisión los principales motivos:

[...] por afecto a un referente homónimo (nombre-homenaje), por costumbre (nombre del santo del día), por mitificación del referente inicial (epónimos), o por el sentido poético del antropónimo (antroponimización de abstractos, fitónimos, etc.). El nombre-homenaje se impone por afecto al modelo, por la necesidad de reconocerlo, conservando el signo que lo identifica o lo identificó. No son patronímicos complementarios sino el homónimo de los padres, también de otros seres queridos, o bien de los lugares que se añoran, no obstante, la predilección de la sociedad por los antropónimos de tradición familiar no ha sido óbice para el avance de tendencias más innovadoras.

Los nombres mitológicos son los portados por personajes que en la elección se declaran admiradores de un referente homónimo, histórico o de actualidad, real o de ficción. Si antaño los modelos se encontraban en el santoral, hoy los adultos han ampliado el campo de búsqueda con las nuevas tecnologías [...]. Hoy interesan mucho más los héroes del cine o de la televisión, de las canchas, de los estadios de fútbol, o los personajes que asoman por Internet, que aquellos que han crecido en la inmensa realidad del laboratorio; desde luego sigue siendo inferior el 
grupo de eruditos que justifica la imposición del antropónimo con datos históricos, aplicando sus conocimientos humanísticos o científicos para este fin.

La difusión de un nombre se acelera cuando el elector iletrado se inspira en homónimos sin recurrir a la información enciclopédica, tal proceso de popularización explica que hoy sean corrientes nombres tomados de la mitología grecolatina o de la literatura e imitados por personas sin conocimientos literarios: Héctor, Lara, Minerva, Nerea, Penélope [...]. Otro grupo lo constituyen nombres transparentes que previamente han recibido un tratamiento metafórico; participan de esta tendencia numerosos simpatizantes del movimiento hippy: Luna, Mar, Nieve, Luz, Sol, Azucena, etc. (2014, p. 34).

Esta autora utiliza en su explicación dos conceptos que tienen una relación entre sí: referente y motivación. Generalmente las motivaciones, entendidas como todas las razones generales que influyen en la elección, tienen un referente al que indican: un familiar, un actor, un personaje, un elemento natural, una deidad, pero en otras ocasiones no. Por tanto todos los refentes constituyen una motivación pero no todas las motivaciones presentan un referente.

Atendiendo a estas características, en este artículo se denomina referente de un nombre, en primer lugar, a aquellos nombres de personas, ya sean paradigmas reales o ficticios, específicos o universales, que sirven de modelo para nombrar a un recién nacido. La tradición ha acuñado, además, determinados sustantivos comunes que han perdido el vínculo con la realidad que nombraban y forman parte de los sistemas antroponímicos.

Estos referentes pueden ser de variada naturaleza, como se evidencia en las ideas aportadas por García Gallarín y los demás investigadores. Se agrupan en la presente investigación en cinco categorías:

1. Familiares: se incluyen en esta categoría los nombres de los padres, abuelos, bisabuelos, tíos, primos, otros familiares más alejados así como padrinos y amistades que por su nivel de relación con alguno de los padres, fundamentalmente, constituyen un referente. 
2. Religiosos: se incluyen en esta categoría referentes bíblicos, nombres incluidos en santorales católicos, nombres de Papas, advocaciones siempre y cuando se declaren como tales, etc.

3. Personalidades: se incluyen nombres de personas vinculadas a las artes, ${ }^{4}$ el deporte, la ciencia, la historia, la política, etc., que constituyen un referente por las habilidades, cualidades o acciones que desarrollan o desarrollaron.

4. Personajes: se incluyen nombres de personajes de obras musicales, teatrales, literarias, cinematográficas, radiales, televisivas, etc., en todas sus formas y manifestaciones.

5. Naturales y geográficos: se incluyen nombres tomados de referentes y fenómenos naturales (arena, sol, luz, rocío, etc.) así como nombres de plantas, piedras preciosas, objetos; además de topónimos vinculados a ciudades, regiones, naciones y continentes.

En la actualidad los antroponomastas ${ }^{5}$ cuentan con varias fuentes de obtención de datos para las investigaciones. Se puede acceder a padrones (Alfaro Gómez, 2010; García Cornejo, 2001; García Gallarín, 2007 y 2014; Gorrotxategi, 2000;), corpus del español de diferentes características (Elsayed, 2018; Simón Parra, 2008), periódicos (Bernárdez, 2016), registros de instituciones: educativas (Reyes y Marrero, 2013; Sabet y Zhang, 2020) y telefónicos (Mora Monroy, 1976), fuentes jurídicas (Harb y Fawwaz, 2016; López Franco, 2008-2009) y religiosas (BoydBowman; 1970, Carretero, 2001).

Los datos pueden aparecer en estadísticas nacionales (Boullon, 2016; Garaio, 2017), muchas de las investigaciones anterio-

\footnotetext{
${ }^{4}$ Este concepto va más allá de las siete bellas artes reconocidas: arquitectura, escultura, pintura, música, literatura, danza y el cine, e incluye a profesionales del teatro, la televisión, la radio, entre otros. No solo se circunscribe a actores, actrices o directores, sino a periodistas, locutores, etc.

${ }^{5}$ Los autores y textos citados en este artículo no constituyen los únicos representantes de las tendencias y líneas de investigación por las cuales se cita. Han sido citados en virtud de que sus trabajos constituyen un ejemplo.
} 
res de carácter onomástico, o no, sirven como fuentes de análisis (Carriet Valiente, 2019; Baez, Herrera y Mendoza, 1993; Jia, 2009; Martínez Nazario, 2016; Ruhstaller, 1993) y hasta en la literatura se encuentra material para la investigación antroponomástica (Coduras, 2003; Herrero Ingelmo, 1996).

Entre tantas posibilidades y acceso a determinadas informaciones relevantes ¿cómo se obtienen los datos para conocer sobre las motivaciones y referentes? La vía más efectiva y utilizada es la encuesta en sus múltiples variantes, pues las demás formas tienen determinados inconvenientes que imposibilitan una confirmación sobre los motivos.

$\mathrm{Al}$ analizar el padrón electoral ${ }^{6}$ madrileño la investigadora García Gallarín logra detectar tendencias a través del análisis formal de los nombres con el objetivo de abordar la evolución de la antroponimia hispanoamericana, no obstante, plantea una limitante: "Sin encuestas es imposible precisar cuáles son los nombres preferidos por los distintos sectores sociales y la verdadera motivación de la elección [...]” (2007, p. 231).

En concordancia con lo anterior Kohoutková sostiene, a raíz de su análisis de una muestra antropónimos españoles, que "los motivos no se pueden detectar con exactitud, pero es probable que se repiten los de la historia: afirmar la protección a los niños, y desear a ellos el mismo destino como tienen actuales portadores del nombre" (2009, p. 20).

\section{Las encuestas y entrevistas en la investigación antroponomástica}

Las encuestas y entrevistas son necesarias, no solo para obtener la información relativa a las motivaciones, sino para otras for-

\footnotetext{
${ }^{6}$ Sobre la valía del padrón como fuente, la propia autora explica: "El padrón es la fuente que nos ha permitido avanzar en el conocimiento de la antroponimia hispanoamericana, identificando los nombres más frecuentes, los generacionales o los característicos de una región o de una etnia; hemos descubierto las huellas onomásticas que dejan los cultos a los santos y los misterios o milagros atribuidos a la Virgen, pero los nombres nos dan también el grado de secularización de un país por los referentes en los que se inspira los designadores, y en general los influencias culturales más directas" (2007, p. 209).
} 
mas de recogida de datos: la recopilación de nombres de pila, la descripción de fenómenos vinculados al uso lingüístico de estos, el registro de actitudes y memorización de determinados nombres propios.

Generalmente las bases de datos tienen un carácter generacional incompleto ${ }^{7}$ o no se puede acceder a ellas, por lo que se utiliza la encuesta como forma de compilar nombres de una o más generaciones de familiares. Bajo estas premisas Abdul Wahed Qasem Ghaleb Al-Zumor (2009) recopiló casi 300 nombres femeninos personales utilizando un cuestionario en varias provincias en Yemen. De igual manera Orlando Alba (2013) utilizó una encuesta para construir el corpus de su investigación. Alba entrevistó a 804 informantes jóvenes (entre 16 y 20 años de edad) para obtener más de cinco mil nombres pertenecientes a tres generaciones distintas de residentes dominicanos. El objetivo fue evidenciar diferencias generacionales y socioculturales en cuanto a la selección del nombre y el uso de hipocorísticos.

En la tradición investigativa es frecuente vincular el estudio de los hipocorísticos, como forma peculiar de uso y transformación de los antropónimos, con la utilización de cuestionarios y entrevistas. Cronológicamente se puede citar la investigación de Mikío Urawa (1985) quien aplicó encuestas a 53 informantes nacidos y residentes en Bogotá, la mayor parte de ellos entre los veinte y los cuarenta años.

Ya en el siglo xxi sobresalen los trabajos de Lucila Gutiérrez Santana (2009-2010 y 2013-2014). Esta investigadora aplicó, primeramente, encuestas a 178 estudiantes de las universidades de Colima (México) y de Concepción (Chile) para analizar la palatalización en algunos hipocorísticos femeninos, más tarde publicó los resultados sobre su análisis de hipocorísticos y

\footnotetext{
${ }^{7} \mathrm{Si}$ se tiene la base de datos de los estudiantes matriculados en una universidad solo se accederá a los datos de una determinada generación, o en los casos más favorables se registrarán, además de los datos del alumno, los de sus padres. Si se accede a los datos del Ministerio de Salud de una determinada nación de igual manera la distribución pudiera diferir de unas décadas a otras, incluso al analizar un padrón electoral no tendríamos los datos de las personas sin derecho a participar en las elecciones y sería imposible vincular, por el parentesco, a las personas registradas en él.
} 
truncamientos de nombres propios indoeuropeos y de la lengua náhuatl. La muestra piloto se aplicó a 12 estudiantes de la Universidad de Colima. Por su parte Miguel Reyes Contreras (2020) entrevistó a 20 personas entre hablantes y no hablantes del mazahua para detectar truncamientos de hipocorísticos españoles y nombres de pila en esta lengua.

Juan Bernardo Estrada (2014) para recopilar y clasificar los mecanismos de formación de hipocorísticos en México preparó un corpus de 204 nombres propios extraídos de varias fuentes (artículo científico, calendario y tesis doctoral). Para la entrevista seleccionó cuatro informantes que hubieran nacido y radicado la mayor parte de su vida en las ciudades objeto de estudio, las cuales se escogieron por representar las zonas dialectales de México.

Carmen Fernández ha utilizado la encuesta como forma de recolección de datos en al menos dos de sus investigaciones en la presente década. En 2008 publica los resultados sobre los sobrenombres o epiclesis dentro del sistema antroponímico, su uso y aplicación dentro de una comunidad semiurbana atendiendo a las variables sexo y edad. Se entrevistaron 72 personas agrupadas en intervalos de 20 años. En 2019, junto a Natividad Hernández Muñoz, realiza un análisis socionomástico de los antropónimos desde los postulados metodológicos de la disponibilidad léxica. La encuesta se aplicó a 60 informantes agrupados atendiendo al género, la edad y los estudios educacionales realizados.

Al ser un elemento de selección, lo nombres están sujetos a fenómenos de aceptación y rechazo popular, generalmente asociados a determinados estratos sociales. Gastón Salamanca y Lidia Pereira (2013) para arribar a conclusiones sobre este particular aplicaron una encuesta a 40 sujetos de nivel educativo superior (universitario) en la que indagan sobre la actitud de prestigio o estigmatización que tenían respecto a 60 nombres propios de persona. Por su parte José Edwin Jacinto Santos (2013-2014) entrevistó a 24 personas para obtener la muestra para la realización del estudio sobre apellidos y nombres propios de personas en la comunidad ashéninka del Gran Pajonal.

Si en las diferentes investigaciones anteriormente descritas la encuesta ha resultado de vital importancia para el logro de los 
objetivos, en los análisis sociolingüísticos sobre los motivos de selección antroponomástica lo es más.

Marlene Arteaga Quintero y Yaritza Cova (2003) aplicaron un cuestionario escrito a 138 estudiantes $^{8}$ en el que se interesaban por los nombres de parientes más cercanos, el lugar de residencia, el gusto, las razones de selección y la posibilidad de cambio de nombre de cada uno de los informantes. La muestra estuvo constituida por estudiantes de los cursos de tres docentes: dos cursos del Instituto Universitario Jesús Obrero de Fe y Alegría (47 estudiantes), dos de la Universidad Católica Andrés Bello (46 estudiantes), y dos del Instituto Pedagógico de Miranda J. M. Siso Martínez (45 estudiantes).

Entre las respuestas dadas por los encuestados están la de haber recibido sus nombres por: el nombre de una protagonista o personaje de novela, de una escritora, los de sus padres, de una actriz, una fragancia, por apellidos de presidentes, por herencia familiar, un grupo explicó que lleva el nombre de un familiar; por artistas de moda, por personajes de telenovelas o por el significado bíblico.

Uno de los estudios más recientes realizados en Latinoamérica sobre motivación corresponde a Fabiola Águila Martínez (2014). La autora recoge, a través de encuestas escritas aplicadas en tres regiones mexicanas, las razones que esgrimen las madres para seleccionar el nombre y organiza los parámetros más utilizados según las clases sociales y el nivel de instrucción de las entrevistadas. Las 450 encuestas fueron cuidadosamente aplicadas de forma equitativa y organizados los informantes según su clase (baja, media, alta), su escolaridad (primaria, secundaria, bachiller, universitaria) y su estado civil (casada, unión libre y soltera).

Al igual que la investigación anterior, el objetivo de la autora es vincular estas motivaciones con factores sociales como

\footnotetext{
${ }^{8}$ En el apartado 2 del artículo se expone que la muestra está conformada por 119 estudiantes. Más adelante se afirma: "la encuesta aplicada a los ciento diecinueve nueve (138) estudiantes, pertenecientes al IUJO (47, cuarenta y siete), UCAB (46, cuarenta y seis) y Siso Martínez (45, cuarenta y cinco)" (p. 19). Se ha optado por utilizar la cifra de 138 por ser esta la suma de todas las encuestas aplicadas según las autoras, pues esos datos no varían en el artículo como sí lo hace la suma total.
} 
el nivel educativo, el estado civil y la clase económica. No obstante, se relacionan las principales respuestas aportadas por las madres. En resumen, eligieron los nombres de sus hijos por preservar la tradición del nombre de su progenitor o del abuelo paterno, con base en el día que nació (santoral), por hacer una promesa a un santo o por el nombre de santo o virgen. Otras tomaron como referente a alguna canción, cantante, flor, pintora, actriz, artista de televisión, jugador de fútbol o baloncesto. Un segundo grupo planteó como razones el significado, por innovar un nombre, por romper la tradición y por la opinión de algún familiar, mientras determinadas madres lo seleccionaron por razones vinculadas con el gusto, la sonoridad o por haberlo leído en un libro o visto en la televisión.

Como se puede apreciar, las respuestas recibidas ${ }^{9}$ concuer- $^{-}$ dan tanto con los principales presupuestos planteados por García Gallarín como con las cinco categorías propuestas para la clasificación de los referentes. Si bien es cierto que con los datos que aportan estas investigaciones, en las que apenas se citan determinados nombres de pila, es difícil identificar el referente exacto; no es complejo determinar a cuales de las categorías pertenece.

Aunque las dos formas de recogida de datos presentadas corresponden a encuestas escritas presenciales, las nuevas tecnologías permiten utilizar otras herramientas. En 2017 Beñat Garaio Mendizabal publicó los resultados de su investigación sobre las motivaciones de padres no vascos para seleccionar nombres vascos a hijos nacidos fuera de la Comunidad Autónoma. Para la obtención de las respuestas se creó un formulario en Google Forms y se contactó con 200 personas a través de Facebook, de las cuales 26 cumplían con las precisiones señaladas. $\mathrm{Al}$ preguntar sobre las motivaciones para seleccionar un nombre vasco las principales respuestas fueron:

"porque a mis padres les gustaba", "porque mi tío quiso llamarme asi” y similares. [...] Mi padre tenía un amigo, vasco, el cual tenía

\footnotetext{
${ }^{9}$ Se obviaron un grupo de respuestas en las que los entrevistados negaban conocer el motivo de su nombre, y en tanto, inservibles para el propósito que sigue este artículo.
} 
una hija que se llamaba Naiara, le gustó el nombre y por eso me puso a mí 'Nayhara". [...] En ocasiones, por otro lado, se pone un nombre vasco simplemente porque "a mi madre le gustó", "porqué [sic] nos gustaba”, "me gustó cuando lo oí por primera vez", "desconocía el nombre, me lo dijeron como posible opción y me gustó", o "por llegar a un acuerdo entre padre y madre" (2017, pp. 70-71).

Como se ha evidenciado, la aplicación de cuestionarios es un eficaz método de recogida de datos pero obtenerlos es solo el primer paso. Las investigaciones sobre las motivaciones y referentes en la selección de los nombres de pila tienen determinadas limitaciones las cuales deben ser conocidas y superadas por el analista.

\section{Límites y particularidades de la investigación motivacional}

Como se ha evidenciado, la principal utilidad de las encuestas radica en la imposibilidad de conocer exactamente cuáles son los motivos o referentes que favorecieron la elección de un nombre de pila sobre el resto solo conociendo el nombre, es decir, solo por su aspecto formal. Si en un directorio telefónico aparecen los nombres David y Emma sería difícil ubicar el referente preciso que sirvió de modelo a menos que se contacte con quien lo lleva, incluso a riesgo de que su portador desconozca el motivo.

David puede ser nombre de un familiar o amigo, puede estar inspirado por el rey de Israel y personaje bíblico, el cantante español (Bisbal), el futbolista inglés (Beckham), el ilusionista o el personaje de la novela homónima de Charles Dickens (ambos Copperfield). De igual forma sería complejo determinar si al nombrar a Emma influyó el gusto por determinadas actrices (Watson, Stone, Roberts, Mackey, etc.), la lectura de la novela homónima de Jane Austen o su adaptación al cine en 1996, el nombre de una vecina e incluso es probable que el motivo provenga del atletismo y el paradigma sea Emma Coburn, campeona mundial de los 3000 $\mathrm{m}$ con obstáculos. Todas estas posibilidades son igual de válidas hasta tanto no se corroboren o descarten. 
Aspectos como la edad de la persona, el país de nacimiento, el origen de sus padres, la fecha de nacimiento, entre otros, funcionan en determinados contextos como elementos a tomar en cuenta para determinar el referente. Sin embargo, sin una entrevista que confirme las teorías, todos los análisis quedarían en el terreno de las suposiciones y las probabilidades.

Otra particularidad derivada de la no relación entre forma y referente es que ambas esferas pueden variar sin que varíe la otra. El cantante brasileño Roberto Carlos fue el referente en que se inspiró el padre del jugador brasileño homónimo para seleccionar el nombre de su hijo, a su vez el astro del futbol nombró a uno de sus hijos de la misma manera. Por tanto, sin variar la forma ha variado el referente: primero es por el cantante y en una segunda denominación es por el padre.

De igual manera puede suceder que varíe la forma y se mantenga el mismo referente: es muy común que los hermanos compartan variantes del nombre de sus padres, o que hijos hereden el nombre de sus padres con determinados cambios que faciliten una identificación. Es posible que múltiples factores culturales, situacionales, ortográficos o de gusto influyan en que se varíe la forma tradicional de escribirse el nombre de un referente. Estas variaciones no constituyen una limitante para validar el vínculo con el referente. ${ }^{10}$

A la multiplicidad de los referentes y su carácter variable se une el hecho de que la clasificación por el referente tiene límites que la hacen no aplicable a un grupo determinado de nombres de pila. Existen nombres que son seleccionados por puro gusto de los padres, por su significado, por cuestiones funcionales más allá de la identificación, por eufonía, entre otros. Estos argumentos constituyen motivaciones válidas; sin embargo, no poseen un referente identificable, y por tanto quedan fuera de la clasificación propuesta.

\footnotetext{
${ }^{10}$ Se han identificado dos formas en las que lingüísticamente se produce la copia del referente: total y parcial. En la primera se calca el referente totalmente o uno de los elementos del compuesto (Anthony por Marc Anthony) mientras que en la segunda se evidencian transformaciones que pueden ser de supresión, de adición, metátesis, sustitución, etc.
} 
Otro factor en contra del investigador es la imposibilidad de dar con el referente a pesar de saber a qué categoría pertenece, como quedó evidenciado anteriormente. Es posible que en la entrevista el informante plantee que su nombre fue extraído de una revista, escuchado en la radio, visto en una novela o leído en un libro, y en consecuencia no poder identificarlo exactamente por falta de información. Hay una alta probabilidad de que sea una persona real si se vio en un noticiero, si se extrajo de una revista, o se eligió de una guía telefónica. De la misma manera, si se indica que fue seleccionado de un libro de aventuras es casi seguro que se trate de un personaje de ficción.

A pesar de todas estas limitaciones es posible identificar en la mayoría de los casos un referente y poder clasificarlo según las categorías propuestas. La teoría y la práctica antroponomásticas se han encargado de identificar las principales tendencias en cuanto a motivaciones y referentes, llegando incluso a vincularlas con múltiples factores, entre ellos los sociales. No obstante, poco se ha dicho sobre las formas en que se vinculan las formas de composición de los nombres de pila y los referentes asociados. Socializar los principales resultados obtenidos en materia de clasificación es el objetivo de los siguientes apartados.

\section{Relación entre referente y estructura en los nombres de pila}

Antes de explicar las relaciones referente-estructura es necesario especificar los criterios por los cuales se clasifican en este trabajo los nombres de pila, puesto que ya se ha explicado la metodología para la clasificación de los referentes.

Los nombres simples son aquellos que están compuestos por un solo elemento (Sofía, Luis, Marcos, etc.) y los compuestos, propiamente dichos, son los conformados por dos elementos tradicionalmente escritos de forma separada. En ocasiones esta distinción se vuelve compleja porque: "a menudo varios nombres de pila se combinan para dar lugar a un antropónimo compuesto, cuya escritura suele mantener la autonomía gráfica de los nombres que lo integran: José Antonio, María de los Llanos, 
Luis Alberto, Elena María [...] han alcanzado cierta extensión e incluso arraigo en el uso de grafía simples para ciertos antropónimos compuestos: Mariángela, Maríaluz (también Mariluz), Juanjosé" (RAE, 2010, pp. 626-627).

Esto conlleva a que la clasificación por el número de palabras no siempre sea efectiva. Por ejemplo Anakarla es de grafía simple pero es un nombre de pila compuesto y posee el mismo valor que la variante más usada y aconsejada Ana Karla. En no pocas ocasiones resulta complejo la delimitación por lo que se ha decidido seguir la propuesta planteada en la Nueva gramática de la lengua española para la composición en general:

Se suelen distinguir tres tipos fundamentales de compuestos: los compuestos propios o univerbales, los compuestos sintagmáticos y los compuestos sintácticos. Los componentes de los primeros se integran en una única palabra ortográfica y, por lo general, en un único grupo tónico: agridulce, drogodependiente [...]. Cuando uno de los dos segmentos, casi siempre el primero, está acortado, se obtienen los llamados compuestos acronímicos, como en cantante + autor $>$ cantautor $(\$ 1.3 .4 \mathrm{~d})$ [...]. Los compuestos del segundo tipo se forman yuxtaponiendo palabras que mantienen su propia independencia gráfica y acentual, unas veces separadas con guion intermedio (árabe-israelí,) y otras sin él (cabeza rapada, casa biblioteca) (RAE y ASALE, 2009, p. 192).

Ajustando estos principios a las características propias de los nombres de pila se tienen dos tipos de nombres compuestos ya que el tercer tipo (compuestos sintácticos) no resulta de nuestro interés. En consecuencia, nombres como Marisol (María + Sol), Mariluz (María + Luz), Osviel (Osvaldo + Daniel) son tan compuestos como María Sol, Luis José y Osvaldo Daniel. Es importante tener en cuenta el valor de las motivaciones en la selección del nombre de pila debido a que no siempre la estructura formal (ver Marisleidy en la muestra) indica la necesaria formación de un nombre compuesto (María + Leidy) como sí ocurre en los sustantivos comunes.

La relación entre las referencias y los elementos que componen los nombres de pila no es unívoca pues un nombre propio 
puede motivarse tanto por uno como por múltiples referentes. ${ }^{11}$ Estos referentes pueden vincularse, en el nombre de pila compuesto, tanto a los elementos de manera independiente como al nombre en su totalidad. Atendiendo a este principio de univocidad se identifican seis esquemas generales de interacción entre elemento y referente:

\section{Nombre de pila simple vinculado a un solo referente (NSR)}

En este apartado se ubican los nombres simples que estén asociados a un único referente que puede ser de estructura simple o compuesta. El vínculo con el referente puede ser mediante la copia total o parcial de algunos de los elementos que lo componen. Ejemplo: Ainhoa por la protagonista de una serie española "El Barco" o Yoel por su padre de igual nombre. ${ }^{12}$

\section{Nombre de pila simple vinculado a múltiples referentes (NSMR)}

Bajo esta denominación se clasifican los nombres simples que estén asociados a múltiples referentes. Estos referentes pueden ser homocategoriales (pertenecen a la misma categoría de las antes presentadas) o heterocategoriales (pertenecen a categorías distintas). Los múltiples referentes pueden coincidir formalmente o ser combinados para conformar el nombre de pila. Ejemplo: Yanela, toma su inicial de sus padres (Yamilet y Yoandi).

\footnotetext{
${ }^{11}$ Como resultado de las investigaciones desarrolladas en la Universidad Central de Las Villas (UCLV) se ha precisado que los nombres de pila, ya sean simples o compuestos, son asociados generalmente con tres referentes distintos como máximo.

${ }^{12}$ Todos los ejemplos fueron tomados de la muestra recogida de la tesis de licenciatura Análisis sociolingüístico de los nombres propios de los nacidos en Santa Clara en 2018 de Evelyn Linet Rabelo Fresno.
} 
Nombre de pila compuesto por elementos vinculados al mismo referente (NCUR)

En este apartado se ubican los nombres compuestos que tiene un referente también compuesto y por tanto los dos elementos que lo integran están asociados al mismo referente. La asociación puede manifestarse de varias formas: copia total o parcial de ambos elementos, el cambio de orden de los elementos e incluso la combinación de variantes de denominación. ${ }^{13}$ Ejemplo: Daylis Bárbara copia exactamente el nombre de su madrina o Javier Alejandro el de su padre.

\section{Nombre de pila compuesto por elementos vinculados a referentes independientes (NCRI)}

En este apartado se ubican los nombres compuestos que estén asociados a referentes independientes aunque pertenezcan a la misma categoría o no. Es posible que los referentes sean familiares del mismo grado de parentesco, pertenezcan al mismo tipo de producto cinematográfico o se vinculen al mismo deporte. Sin embargo se trata de referentes independientes a diferencia de lo que sucede con los NCUR analizados anteriormente. Ejemplo: Samantha de la Caridad, el primero por el personaje de la película "Sex and the City", y el segundo por la advocación mariana de la Virgen de la Caridad del Cobre o Danel Enrique, el primero por la inicial de su madre (Danna) y el segundo es el nombre del padre.

\section{Nombre de pila compuesto por elementos vinculados a referentes independientes y múltiples (NCRIM)}

Se clasifican como tales los nombres donde uno de los elementos tiene un único referente mientras el otro elemento es asociado a

\footnotetext{
${ }^{13}$ Existen varias formas de denominación que pueden referirse a una misma persona. Para la investigadora Noroña Vila estas pueden ser: "el nombre de pila, el patronímico, el apellido, el apodo, el seudónimo, el nombre clandestino y el sobrenombre” (1985, p. 290).
} 
múltiples referentes. Es posible que uno de los referentes múltiples esté asociado al elemento que mantiene la conexión referencial única. La diferencia con los NCRI es que los elementos que los componen tienen una conexión independiente y única con su referente y los NCRIM tienen uno de los elementos con un vínculo referencial múltiple. Ejemplo: Christopher Alejandro tomó su nombre del cantante Christopher Alexander y el segundo elemento fue modificado para que coincidiera con el nombre del padre (Alejandro).

Nombre de pila compuesto por elementos vinculados a múltiples referentes (NCMR)

Aquí se incluyen los nombres compuestos cuyos elementos están asociados a múltiples referentes los cuales pueden coincidir o no. La diferencia con los NCRIM es que en los clasificados de esa manera poseen un elemento con un solo referente mientras que los NCMR ambos elementos tiene que estar asociados al mismo tiempo a dos o más referentes.

Además de estos seis grupos clasificatorios, según la relación entre estructura y referente se identifican otros dos como consecuencia de los límites que tiene la clasificación según el referente. Estos otros dos grupos surgen de los NCRI y NCMR y constituyen variantes incompletas al no identificarse el o los referentes de uno de los compuestos.

Nombre de pila compuesto por un elemento sin referente reconocible y otro independiente (NCSRI)

En esta categoría se agrupan los nombres estructurados por dos elementos de los cuales uno no tiene un referente, aunque tenga una motivación, mientras el otro elemento posee un referente único. Cualquiera que sea el orden de los elementos mientras cumpla con estos requisitos el nombre es clasificado como NCSRI. Ejemplo: Lizz Adriana tomó su segundo nombre por su padre (Adrián) y el primero por gusto de sus padres. 
Nombre de pila compuesto por un elemento sin referente reconocible y otro múltiple (NCSRM)

Como NCSRM se clasifican los nombres de pila que estructurados igualmente que NCSRI tienen una diferencia con estos: el elemento que posee referencialidad la establece con dos o más referentes al mismo tiempo.

Este sistema categorial aplicado a un corpus de nombres propios basados en los referentes que motivaron su elección es una herramienta útil para la investigación antroponomástica. La posibilidad de analizar diacrónicamente estos indicadores se une a las tendencias tradicionales de analizar la evolución de los nombres por su forma, sus usos, las motivaciones que los originan, entre otros.

\section{Aplicación a una muestra}

Con el objetivo de comprobar la eficacia de las categorías antes propuesta se aplicó una encuesta escrita a 80 estudiantes matriculados en la Universidad Central "Marta Abreu" de Las Villas elegidos aleatoriamente. Se seleccionaron los informantes pertenecientes a esta institución debido a que en ella matriculan estudiantes de al menos cinco provincias del centro de Cuba, lo que hace la muestra mucho más variada. El estar vinculados docentemente a la mencionada institución y radicar en ella el grupo de investigación sobre onomástica del cual formamos parte, facilitó la recogida y el análisis de los datos.

Para tener resultados compatibles con futuros análisis todos los entrevistados nacieron en el mismo año: 2001. Debido a la disparidad numérica entre el sexo de los informantes (de los 45 que ofrecieron una motivación solo 3 son varones) fue imposible realizar un análisis atendiendo a la variación sexual.

De las 80 respuestas obtenidas, $6(7.5 \%)$ no aportaron ningún dato relevante por desconocer los portadores el motivo o el referente que influyó en la selección de sus nombres de pila. Además, un total de 29 (36.25\%) ofrecieron argumentos vinculados con motivaciones no referenciales como gusto personal, 
propuestas y disputas familiares, razones de singularidad de los nombres dada su rareza, entre otros. Lo que da un total de 45 (56.25\%) respuestas con valor identificativo.

Al comparar los resultados de otras investigaciones realizadas $^{14}$ en la UCLV se ha llegado a la conclusión de que hay mayores posibilidades de obtener referentes cuando se entrevista a los padres sobre el origen del nombre de sus hijos que si se les pregunta a estos. Cuanto menor sea el tiempo trascurrido entre el momento de la selección y el de la entrevista, más alta será la exactitud de la información. Cuando la entrevista es a quienes llevan el nombre, muchos alegan el gusto de los padres, sin más detalles o se concentran en informar de quién fue la idea. Estos factores pudieron influir en que solamente el 56\% de los entrevistados identificara algún referente. Es posible que con mayores recursos y tiempo el cambio de una encuesta escrita a una entrevista aumente las estadísticas de identificación.

A continuación, se muestran las 45 respuestas clasificadas según las ocho categorías propuestas. Los datos ofrecidos corresponden a la información brindada por cada entrevistado. En ocasiones, especialmente con los referentes cinematográficos y musicales, se han añadido algunos datos.

\section{Nombre de pila simple vinculado a un referente (NSR)}

- Cristina: por el nombre de su madre

- Marisleidys: para que se pareciera al de su madre (Marilis)

- Ilianet: para que conservara la inicial del padre (Ismael)

- Rosmery: para que se pareciese al de su hermana (Roxana)

- Amelia: por el nombre de su abuela materna

\footnotetext{
${ }^{14}$ Según resultados preliminares (mayo de 2020) de investigaciones desarrolladas por los autores, existen dos factores que influyen en los niveles de identificación de los referentes: el tamaño de la muestra y los informantes. En una encuesta aplicada a más de mil informantes coetáneos con los informantes aquí reseñados se obtuvo un $72.1 \%$ identificación. En el otro sentido, en una muestra de 250 informantes padres de los niños, se obtuvo un $83.2 \%$.
} 
- Anabel: para que tuviese la misma letra inicial que su hermano (Andrés)

- Roxany: por el nombre de una prima (Rosany)

- Maivy: por la hija de un amigo que tenía ese mismo nombre

- Laura: por una alumna de su madre de igual nombre

- Breisy: por el nombre de una vecina

- Elianis: por la protagonista de una novela ${ }^{15}$

- Jennifer: por la cantante y actriz estadounidense de origen puertorriqueño Jennifer López

- Adianez: por una locutora de radio

- Daniela: por una cantante, presumiblemente Daniela Romo

- Thalia: por la cantante y actriz mexicana Ariadna Thalía conocida como Thalía

- Harly: por el nombre de una protagonista de una película

- Melissa: por el personaje de la telenovela colombiana Aguas Mansas transmitida en Cuba en 1998

- Ariadna: por la hija de Minos y Pasífae, famosa por ayudar a Teseo a salir del laberinto del Minotauro

- Lorena: por el nombre de una persona que escucharon en la radio

- Chanel: por la casa de modas francesa homónima que lleva el apellido de su fundadora Coco Chanel

Nombre de pila simple vinculado a múltiples refentes (NSMR)

- Diamela y Dianela (gemelas): surgen de la combinación del nombre de una prima (Diamelis) y la madre (Diana)

- Magdianis: es el nombre de una persona que escucharon en la radio y se asemeja al de su madre (Magalis)

\footnotetext{
${ }^{15}$ En aquellos nombres vinculados a referentes religiosos, personalidades y personajes, en los cuales no se aporten más datos la causa es la falta de información brindada por el informante que impide una identificación fiable del referente. Estas limitaciones pueden llegar incluso a alcanzar tal grado de ambigüedad, como el caso señalado, que no se precisa si la protagonista es de una novela como género literario o producto televisivo.
} 
Nombre de pila compuesto por elementos vinculados al mismo referente (NCUR)

- María de los Ángeles: por el nombre de su madre

- Liliam Laura: por ser el nombre de una persona mencionada en un programa de radio

- Arasay Arahí: por un personaje de una serie de televisión

\section{Nombre de pila compuesto por elementos vinculados} a referentes independientes (NCRI)

- Yeilis Caridad: el primero por parecerse al de su madre (Yenlis) y el segundo por la Virgen de la Caridad del Cobre, advocación mariana

- Amalia Isabel: el primero por una tía de su abuela (María Amalia) y el segundo por su gemela (María Isabel)

- Martha Alejandra: el primero por el nombre de su abuela paterna y el segundo por el de la abuela materna

- Gelvys Miguel: el primero para que se pareciera al de su hermana (Geidy) y el segundo por el nombre del abuelo

- Amanda Alegna: el primero por un personaje de una novela y el segundo por el nombre de la abuela (Ángela al revés)

- Claudia Beatriz: el primero por una cantante y el segundo por el nombre de una tía

- Malena de Jesús: el primero por ser la abreviatura de María Magdalena y el segundo por Jesús, ambos de origen bíblico

- Litzy María: el primero por el nombre de una persona que salió en una revista y el segundo por el nombre de una de las abuelas

Nombre de pila compuesto por elementos vinculados a múltiples referentes (NCMR)

- María Marla: según la informante es tradición familiar ponerle nombres con la letra inicial $\mathrm{M}$ a las mujeres. Cada elemento del nombre de pila tiene múltiples referentes, los cuales, en este caso, coinciden, pues son los familiares del sexo femenino los que fueron nombrados con la letra $\mathrm{M}$ 
Nombre de pila compuesto por un elemento sin referente reconocible $y$ otro independiente (NCSRI)

- Oscar Luis: el primero por el nombre del padre (Oscar)

- Teresa Lisset: el primero por el nombre de la abuela materna

- Melissa Beatriz: el primero por el personaje de la telenovela colombiana Aguas Mansas transmitida en Cuba en 1998

- Melodis Mari: el primero por el personaje animado, hija de Ariel y Eric, presente en la película Ariel 2: el regreso al mar, estrenada en el 2000

- Inge de Liz: el primero por la nadadora holandesa Inge de Bruijin ganadora de tres medallas de oro y una de bronce en los Juegos Olímpicos Sidney 2000

- Javier Eduardo: el segundo por el nombre del padre (Eduardo)

- Gretel Irene: el segundo por el nombre de la bisabuela

- Rachel de la Caridad: el segundo por la Virgen de la Caridad del Cobre

- Zeinab Manuela: el segundo por el nombre de la bisabuela

- Juliet María: el segundo por el nombre de la hermana mayor

De las ocho categorías propuestas se utilizaron seis, pues no se encontró ningún nombre de pila que pudiera ser clasificado como NCRIM o NCSRM. Dos condiciones pueden haber influido en estos resultados:

De las 45 respuestas con valor clasificatorio, 23 (51\%) corresponden a nombres estructurados por un solo elemento, y 22 (49\%) por dos, por tanto la distribución en cuanto a estructura es similar. No obstante, se evidencia (gráfico 1) un predominio de los nombres simples con un solo referente seguido por los nombres compuestos donde se identifican referentes independientes en al menos uno de los elementos que lo conforman (ya que en el caso de los NCSRI uno de ellos responde a motivaciones no referenciales). 


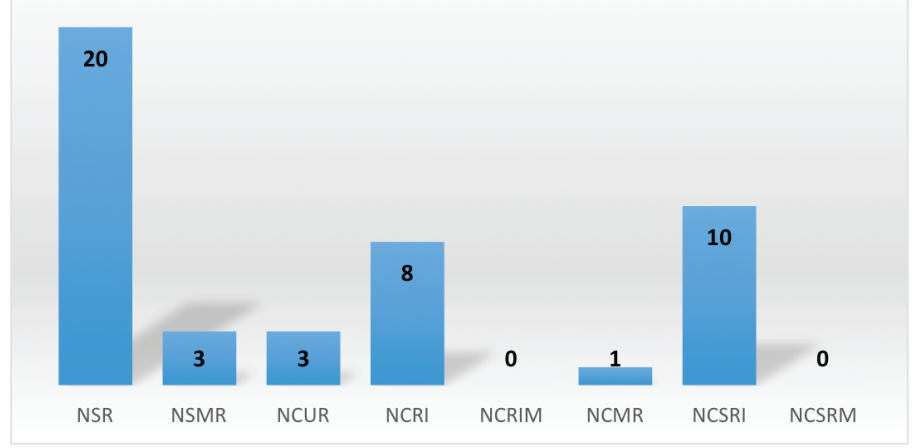

Gráfico 1. Cantidad de nombres según la clasificación motivacional basada en estructura-referente. Fuente: elaboración propia

Al analizar el comportamiento por separado (gráficos 2 y 3 ) se obtiene que casi nueve de diez nombres simples se eligen teniendo en cuenta un único referente y casi ocho de diez (si se suman NCSRI Y NCRI) nombres compuestos con elementos motivacionalmente referenciales están asociados también con un solo referente.

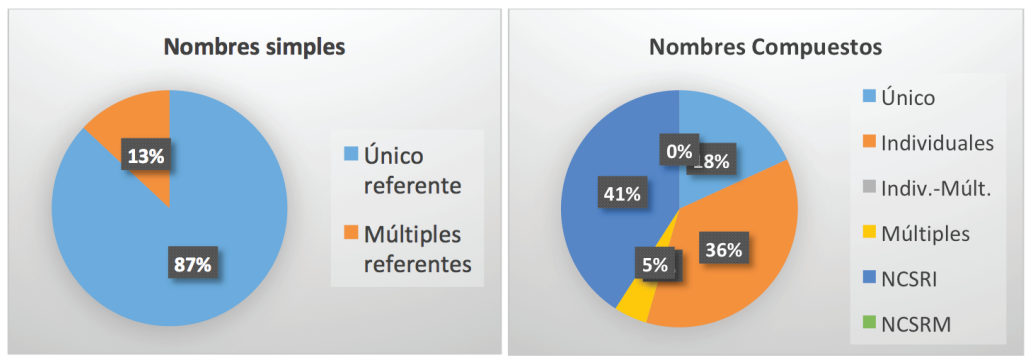

Gráficos 2 y 3. Distribución porcentual de los nombres simples y compuestos según clasificación motivacional basada en estructura -referente. Fuente: elaboración propia

El objetivo del artículo no es investigar las formas de referencialidad en Cuba sino proponer un sistema clasificatorio, el que fue aplicado a una pequeña muestra. No obstante, atendiendo a los anteriores datos se puede afirmar que la tendencia general en los niños cubanos nacidos a inicio del siglo XxI es a 
nombrarlos utilizando un único referente. Autores como Sánchez, García y Díaz (1985) han identificado el gusto por un solo referente en trabajos del siglo $\mathrm{xx}$.

\section{Consideraciones finales}

Como ha quedado evidenciado es factible el estudio de las motivaciones de los nombres de pila a través de la clasificación propuesta. Con los cuestionarios y entrevistas como vías de recolección y tomando en cuenta las limitaciones que tiene este tipo de estudios es posible describir las complejas relaciones que se establecen entre la estructura del nombre de pila y los referentes que los motivan. Aún queda mucho por investigar sobre las relaciones que se establecen entre los tipos de referentes y los mecanismos lingüísticos que se utilizan para la copia, así como el efecto que producen las variables sociolingüísticas en cada uno de estos indicadores. La clasificación aquí presentada, aunque efectiva y mejorable, es solo un primer acercamiento al fenómeno motivacional en los nombres de pila.

\section{Referencias}

Abdul Wahed Qasem Ghaleb Al-Zumor (2009). A SocioCultural and Linguistic Analysis of Yemeni Arabic Personal Names. GEMA: Online Journal of Language Studies, 9(2), 1527. Recuperado de http://journalarticle.ukm.my/2305/

Águila Martínez, F. (2014). Parámetros para la elección del nombre de una persona (tesis inédita de licenciatura). Universidad Autónoma del Estado de México, Estado de México, México. Recuperado de http://sedici.unlp.edu.ar/ handle/10915/5329

Alba, O. (2013). Nombres propios de persona en la República Dominicana. Santo Domingo: Búho. Recuperado de https://scholarsarchive.byu.edu/cgi/viewcontent. cgi article $=1005 \&$ context $=$ books

Alfaro Gómez, E. L. (2010). Dinámica antroponímica y estructura demogenética en Casabindo: siglos XVI al XXI (tesis in- 
édita de doctorado). Universidad Nacional de La Plata, La Plata, Argentina. Recuperado de http://sedici.unlp.edu.ar/ handle/10915/5329

Arteaga, M. y Cova, Y. (2003). Un estudio de onomástica descriptiva. ¿Qué sucede con los nombres propios en Venezuela? Letras, 66, 74-89. Recuperado de https://www.academia. edu/8421709/Un_estudio_de_onom\%C3\%A1stica_descriptiva._Qu\%C3\%A9_sucede_con_los_nombres_propios_en_Venezuela_Coautor\%C3\%ADa_Cova_Yaritza_

Baez, G., Herrera, M. E. y Mendoza, J. F. (1993). Antropónimos en el español de la ciudad de México. Tradición y modernidad. Anuario de Letras, XXXI, 431-496. Recuperado de https://dialnet.unirioja.es/servlet/articulo?codigo $=31500$

BAllester, X. (1999). Contribución a una teoría de los antropónimos. Aion. Annali di Dipartamento di Estudi del Mondo classico e del Mediterraneo Antico, 21, 43-56. Recuperado de https://www.academia.edu/6570538/Contribuci\% C3\%B3n_a_una_Teor\%C3\%ADa_de_los_Antrop\%C3 $\%$ B3nimos

Bernárdez López, A. S. (2016). Historia y traducción: la recepción de los nombres propios de Grecia, China y Rusia en la prensa española del siglo $\mathrm{xx}$ (tesis inédita de maestría). Universidad de Valladolid, Valladolid, España. Recuperado de https://pdfs.semanticscholar.org/6523/74e8ce38f340 da2b332c0a036f3986238206.pdf

Boullon Agrelo, A. (2016). Os nomes galegos no século XXI. En M. González (ed.), Lingua, pobo e terra. Estudos en homenaxe a Xesús Ferro Ruibal. (191-212). Santiago de Compostela: Xunta de Galicia. Recuperado de https://www.academia. edu/31061659/Os_nomes_galegos_no_s\%C3\%A9culo_XXI

Boyd-Bowman, P. (1970). Los nombres de pila en México desde 1540 hasta 1950. Nueva Revista de Filología Hispánica, 19(1), 12-48. Recuperado de https://nrfh.colmex.mx/index.php/nrfh/article/view/437

CAmpo, L. R. (2020). Necesidad de una política lingüística para la normalización de la construcción y escritura de los nombres de pila. Análisis del modelo jurídico cubano desde la perspectiva comparada. Islas, 62(195), 78-97. 
Carretero Melo, A. (2001). Antroponimia en la edad moderna. El nombre de pila del varón. Nouvelle revue d'onomastique, 37-38, 43-56. Recuperado de https://www.persee.fr/doc/ onoma_0755-7752_2001_num_37_1_1396

Carriet Valiente, E. (2019). Los nombres patronímicos en la lexicografía académica. En D. Azorín, Clavería, G. y Jiménez Ríos, E. (eds.), EULA: diccionario de la Academia y su tiempo: lexicografía, lengua y sociedad en la primera mitad del siglo (pp. 253-284). Anexo 5. Recuperado de http://rua. ua.es/dspace/bitstream/10045/100816/1/ELUA-AnexoV_11.pdf

Coduras Bruna, M. (2003). La antroponimia en los libros de caballerías españoles: el ciclo amadisiano (tesis inédita de doctorado). Universidad de Zaragoza, Zaragoza, España. Recuperado de http://zaguan.unizar.es/record/12557\#

Elsayed Mahmoud, R. (2018). La presencia del arabismo en la antroponimia hispánica contemporánea (tesis inédita de doctorado). Universidad Complutense de Madrid, Madrid, España. Recuperado de https://eprints.ucm.es/49361/1/ T40256.pdf

Fernández Juncal, C. (2008). Patrones sociolingüísticos de la onomástica. Revista Española de Lingüística, 38(2), 5-20. Recuperado de https://dialnet.unirioja.es/servlet/articulo?codi go $=3115667$

Fernández Juncal, C. (2011). El sistema antroponímico como diasistema. Nouvelle revue d'onomastique, 53, 141-150. Recuperado de https://www.persee.fr/doc/onoma_0755-7752_2011_num_53_1_1730

Fernández Juncal, C. y Hernández Muñoz, N. (2019). Disponibilidad léxica y socionomástica. Ogigia. Revista Electrónica de Estudios Hispánicos, 25, 185-210. Recuperado de https://revistas.uva.es/index.php/ogigia/article/ view/2916/2881

Garaio Mendizabal, B. (2017). ¿Y cómo llamamos a nuestra hija?: sobre la proliferación de nombres de origen vasco en jóvenes no residentes en la CAV y Navarra. Sancho el Sabio, 40, 57-91. Recuperado de https://dialnet.unirioja.es/ servlet/articulo? codigo $=6233173$ 
García Cornejo, R. (2001). La antroponimia masculina en un padrón de cuantías de 1436. Anuario de Estudios Filológicos, 24, 137-152. Recuperado de https://dialnet.unirioja.es/ servlet/articulo? codigo $=59011$

García Gallarín, C. (2007). La evolución de la antroponimia hispanoamericana. En C. García (comp.), Los nombres del Madrid multicultural (pp. 209-235). Madrid: Parthenon. Recuperado de https://dialnet.unirioja.es/servlet/ articulo? codigo $=4969088$

García Gallarín, C. (2014). Diccionario histórico de nombres de América y España. Madrid: Sílex. Recuperado de https:// dialnet.unirioja.es/servlet/libro? codigo $=556278$

Gorrotxategi Nieto, M. (2000). Evolución del nombre de pila en el país vasco. Revista Fontes Linguae Vasconum: Studia et Documenta, 83, 151-168. Recuperado de https://dialnet. unirioja.es/servlet/articulo? codigo $=26314$

Gutiérrez Santana, L. (2009-2010). Palatalización en algunos hipocorísticos femeninos de Chile y México. Géneros. Revista de Investigación y Divulgación sobre los Estudios de Género, 16(6), 87-108. Recuperado de http://revistasacademicas.ucol.mx/index.php/generos/article/view/515/pdf

Gutiérrez Santana, L. (2013-2014). Hipocorísticos y truncamiento de nombres propios indoeuropeos y de la lengua náhuatl. Revista Científica de Investigaciones Regionales, 36(1), 73-84. Recuperado de https://dialnet.unirioja.es/ servlet/articulo? codigo $=4675899$

Harb Al-Qawasmi, A. y Fawwaz Al-Abed, A. (2016). A Sociolinguistic Study of Choosing Names for Newborn Children in Jordan. International Journal of English Linguistics, 6(1), 177-186. Recuperado de http://www.ccsenet.org/journal/ index.php/ijel/article/view/56995

Herrero Ingelmo, M. C. (1996). La elección de los nombres propios en Longo. Habis, 27, 157-169. Recuperado de https: //dialnet.unirioja.es/servlet/articulo? codigo $=58058$

Jacinto Santos, P. (2013-2014). Onomástica: Estudio sobre apellidos y nombres de personas en la comunidad ashéniinka del Gran Pajonal (tesis inédita de maestría). Universidad Pompeu Fabra. Barcelona, España. Recuperado de https://www.acade 
mia.edu/4689855/_Onom\%C3\%A1stica_Estudio_sobre_ Apellidos_y_Nombres_Propios_de_Personas_en_la_Co munidad_Ash\%C3\%A9ninka_del_Gran_Pajonal

JiA, L. (2009). Estudio lingüístico sobre los nombres propios de persona en chino (tesis inédita de doctorado). Universidad de Granada, Granada, España. Recuperado de https://doc player.es/42650731-Estudio-linguistico-sobre-los-nombrespropios-de-persona-en-chino.html

Koнoutкová, H. (2009). Los antropónimos españoles con especial atención a los nombres de pila (tesis inédita de licenciatura). Universidad Masaryk, Brno, República Checa. Recuperado de https://is.muni.cz/th/180863/ff_b/bakalars ka_prace.pdf

López Franco, Y. (2008-2009). Mirada lexicológica a la atribución de los nombres de pila en Tlalnepantla de Baz, Estado de México, 1935-1955. Multidisciplina, 2, 17-24. Recuperado de http://www.journals.unam.mx/index.php/multidisciplina/article/view/27686

Martínez Nazario, M. (2016). Antecedentes antroponímicos en la creación de nombres de pila en Puerto Rico. Alborada, 11(1), 47-50. Recuperado de https://www.researchgate.net/ publication/282355792_Antecedentes_antroponimicos_ en_la_creacion_de_nombres_de_pila_en_Puerto_Rico

Mora Monroy, S. V. (1976). Breve estudio sobre apellidos y nombres propios de persona en Colombia. Thesaurus: Boletín del Instituto Caro y Cuervo, 31(3), 536-560. Recuperado de http://bibliotecadigital.caroycuervo.gov.co/533/

Noroña Vila, M. (1985). Aplicación de una terminología onomástica. Anuario L/L, 16, 289-299.

Rabelo Fresno, E. L. (2020). Análisis sociolingüístico de los nombres propios de los nacidos en Santa Clara en 2018 (tesis inédita de licenciatura). Universidad Central de Las Villas, Santa Clara, Cuba.

Real Academia Española (2010). Ortografía de la lengua española. Madrid: Espasa Libros.

Real Academia Española y Asociación de Academias de la Lengua Española (2009). Nueva gramática de la lengua española. Madrid: Espasa Libros. 
Reyes Contreras, M. (2020). Truncamientos de hipocorísticos españoles y nombres de pila en mazahua: un fenómeno compartido. Onomástica Desde América Latina, 1(1), 141-167. Recuperado de http://e-revista.unioeste.br/index. php/onomastica/article/view/24164

Reyes Díaz, M. y Marrero Pulido, J. (2013). Antropónimos. Análisis de una muestra canaria de los dos últimos decenios. Nouvelle Revue d'Onomastique, 55, 191-231. Recuperado de https://www.persee.fr/doc/onoma_0755-7752_2013_ num_55_1_1781

Ruhstaller, S. (1993). Los elementos constituyentes de la antroponimia hispánica y su contenido semántico y referencial. Cauce: Revista Internacional de Filología, Comunicación y sus Didácticas, 16, 131-140. Recuperado de https:// dialnet.unirioja.es/servlet/articulo? codigo $=87731$

Sabet, P. y Zhang, G. (2020). First Names in Social and Ethnic Contexts: A Socio-Onomastic Approach. Language \& Communication, 70, 1-12. Recuperado de https://www.science direct.com/science/article/pii/S0271530919302630

Salamanca, G. y Pereira, L. (2013). Prestigio y estigmatización de 60 nombres propios en 40 sujetos de nivel educacional superior. Universum, 28,2, 35-57. Recuperado de https:// scielo.conicyt.cl/pdf/universum/v28n2/art_03.pdf

Sánchez, M., García, H. y Díaz Gamez, M. (1985). Tendencia de los nombres propios de persona en Santa Clara, 19771981. Islas, 137-166.

Simón PARra, M. (2008). El nombre de persona en la documentación castellana medieval (tesis inédita de doctorado). Universidad de Alcalá, Alcalá de Henares, España. Recuperado de https://dialnet.unirioja.es/servlet/tesis? codigo=79546

Urawa, M. (1985). Muestra de hipocorísticos en el español bogotano. Thesaurus: Boletín del Instituto Caro y Cuervo, 40(1), 51-102. Recuperado de http://bibliotecadigital.caro ycuervo.gov.co/638/ 